\title{
Assessment of the potential health benefits of certain total extracts from Vitis vinifera, Aesculus hyppocastanum and Curcuma longa
}

\author{
DENISA MARGINA $\breve{1}^{1}$, OCTAVIAN TUDOREL OLARU ${ }^{2}$, MIHAELA ILIE $^{3}$, \\ DANIELA GRĂDINARU ${ }^{1}$, CLAUDIA GUȚU ${ }^{3}$, SORINA VOICU ${ }^{4}$, ANCA DINISCHIOTU $^{4}$, \\ DEMETRIOS A. SPANDIDOS ${ }^{5}$ and ARISTIDIS M. TSATSAKIS ${ }^{6}$
}

\author{
Departments of ${ }^{1}$ Biochemistry, ${ }^{2}$ Botany and Cellular Biology and ${ }^{3}$ Toxicology, Faculty of Pharmacy, \\ Carol Davila University of Medicine and Pharmacy, Bucharest 020956; ${ }^{4}$ Department of Biochemistry and Molecular Biology, \\ Faculty of Biology, University of Bucharest, Bucharest 050095, Romania; ${ }^{5}$ Department of Clinical Virology, \\ School of Medicine and ${ }^{6}$ Department of Forensic Sciences and Toxicology, \\ Faculty of Medicine, University of Crete, Heraklion 71003, Greece
}

Received August 5, 2015; Accepted September 2, 2015

DOI: 10.3892/etm.2015.2724

\begin{abstract}
A number of recent studies have illustrated the active role of food/natural components in the prevention of chronic diseases and in the improvement of the quality of life. In the present study, we aimed to obtain and characterize certain extracts from Vitis vinifera L., Aesculus hippocastanum L. and Curcuma longa $\mathrm{L}$., focusing on their antioxidant effects in vitro. Three vegetal extracts were obtained for each plant: in water, $50 \%$ water-alcohol and in $96 \%$ ethanol. These extracts were then analyzed for their qualitative composition by high performance thin layer chromatography (HPTLC) and total phenolic content by ultraviolet-visible spectrophotometry (UV-VIS). The antioxidant activity of the extracts was assessed in vitro by 2,2-diphenyl-1-picrylhydrazyl (DPPH) assay; the effects of lipid peroxidation on the cell membrane were evaluated using Jurkat cells in two experimental models: normoglycemic and hyperglycemic medium, in order for the results to be able to be translated into clinical practice. In addition, the resistance of the extracts to acid and alkaline hydrolysis was investigated. The obtained extracts had 0.4-39 $\mu \mathrm{g}$ phenolics/mg total extract. The largest amount of phenolics was found in the Cucurma longa extracts, while the lowest was found in the Aesculus hippocastanum extacts. HPTLC analysis identified the main phenolic compounds in the extracts which were ferulic acid, gallic acid, caffeic acid and coumaric acid, as well as quercetin, kaempferol,
\end{abstract}

Correspondence to: Dr Denisa Margina, Department of Biochemistry, Faculty of Pharmacy, Carol Davila University of Medicine and Pharmacy, 6 Traian Vuia St., Bucharest 020956, Romania

E-mail: denisa.margina@gmail.com

Abbreviations: DPPH, 2,2-diphenyl-1-picrylhydrazyl, DPPP, diphenyl-1-pyrenylphosphine; HPTLC, high pressure thin layer chromatography; $\mathrm{CuOOH}$, cumene hydroperoxide; FCS, fetal calf serum; PBS, phosphate-buffer saline

Key words: antioxidant effects, vegetal extracts, hyperglycemia apigenin, curcumin, luteolin and esculetin. The Aesculus hippocastanum extracts had a low antioxidant efficacy, while both the Curcuma longa and Vitis vinifera extracts had a high antioxidant activity; the products resulting from alkaline hydrolisis were significantly more efficient in scavenging DPPH radicals compared to the products resulting from acid hydrolisis. The antioxidant effects of the Curcuma longa extracts exerted on the membranes of Jurkat cells were the most prominent under both normal and hyperglycemic conditions. The results of the present study may be translated into clinical practice and demonstrate that Curcuma longa extracts may be effective in both the prevention of diabetes mellitus and in attenuating the development of complications associated with the disease.

\section{Introduction}

Recent research, as well as public opinion have focused on the health benefits of a diet rich in natural products and in the benefits of vegetal supplements. The consumption of natural foods and vegetal supplements or drugs based on natural compounds has significantly increased over the past $10-15$ years. Epidemiological studies have demonstrated the active role of food/natural components in the prevention of chronic diseases, such as cardiovascular diseases or malignant diseases, or even in the improvement of the longevity and quality of life (1-6). The Mediterranean diet, as well as the French paradox, prove that flavonoids, including red wine polyphenols, have the ability to reduce the incidence of cardiovascular disease, even in a population consuming a lipid-rich diet.

Curcuma longa $\mathrm{L}$., Vitis vinifera $\mathrm{L}$. and Aesulus hippocastanum L. species are well-known for their health protective properties. European Medicines Agency presents monographs regarding the quality of the plant materials and chemical composition for the three vegetal products (7-9).

Studies regarding the effects of curcumin were initially conducted due to the popularity of turmeric (Curcuma longa), an aromatic product used in several countries in Southeast Asia which is extensively cultivated in India, China, Taiwan, 
Sri Lanka, Bangladesh, Indonesia, Peru, Australia and the West Indies (10-13). Many species in this rhizomatous genus, Curcuma from the family Zingiberaceae, have been cultivated, and their powdered rhizomes have been widely used in native cooking to provide flavor or as ingredients in traditional remedies. The name Curcuma was established by Linnaeus in 1753, and it is derived from the Arabic word 'kurkum', meaning yellow color, identifying the color of the rhizomes (14). Preclinical studies have proven that curcuminoids, the natural polyphenols extracted from the rhizome of turmeric, have antioxidant, anti-inflammatory and antitumor effects (15-19).

Aesculus hippocastanum L. (Hippocastanaceae) is a large tree, commonly known as horse chestnut. Horse chestnut seeds have been used traditionally for healing purposes for several centuries. The extracts from the seeds are constituents of numerous preparations used both internally and externally for the treatment of patients with chronic venous insufficiency or other inflammatory conditions of the veins, lower leg varicose veins and hemorrhoids, as well as for the treatment of frostbite, burns, epidermal abrasions and skin inflammation (20).

Vitis vinifera leaves are by-products in the wine industry and are used in traditional medicine for their liver-protective, spasmolytic and vasorelaxant effects (21). Owing to their chemical composition (phenolic acids, flavonoids, procyanidins, tannins and vitamins), the leaves are present in dietary supplements with antioxidant properties (22).

The aim of the present study was to obtain different extracts from Vitis vinifera L., Aesculus hippocastanum L. and Curcuma longa L. available on the Romanian market, using solvents with the lowest possible toxicological outcome (purified water and ethanol either 50 or $96 \%$ ). The obtained extracts were characterized for their chemical composition by high performance thin layer chromatography (HPTLC)/ultraviolet(UV) methods.

Considering the fact that natural products undergo significant changes when passing through the digestive tract (acid and alkaline $\mathrm{pH}$, respectively), the behavior of the extracts under acid and alkaline hydrolytic conditions was evaluated, in order to establish whether their antioxidant and protective effects are due to the total content of the extracts or due to their metabolic products. The antioxidant effects of the total extracts, as well as those of their hydrolytic products were evaluated in two different experimental models: their antioxidant activity in vitro was evaluated using 2,2-diphenyl-1-picrylhydrazyl (DPPH) assay and their protective effects against lipid peroxidation in Jurkat cells were assessed using the diphenyl-1-pyrenylphosphine (DPPP) fluorescent probe. Considering the fact that data have demonstrated the ability of natural product-based diets to reduce the incidence of cardiovascular disease (23-26), we also examined the antioxidant and anti-inflammatory [through the intercellular adhesion molecule-1 (ICAM-1) level] effects of the extracts on Jurkat cells exposed to hyperglycemia, a cell model that mimics the in vivo conditions associated with diabetes mellitus, a syndrome known to induce atherosclerosis and cardiovascular disease.

Our results revealed that the extracts were resistant in artificial gastric and intestinal medium and that they release their aglycons only under severe hydrolytic conditions (1 M $\mathrm{HCl}$ or $\mathrm{NaOH}$ under reflux). The biological effects of the extracts were evaluated and compared to those of other known antioxidants, such as ascorbic acid $(10 \mu \mathrm{M})$, curcumin and quercetin (positive controls); the results revealed that the aqueous extracts were poor in flavonoids and exerted a mild antioxidant effect. The ethanol extracts exerted significant antioxidant effects compared to the positive controls, in both experimental models. The Curcuma longa extracts were much more efficient in counteracting lipid peroxidation, particularly under pathological conditions and exerted greater protective effects against oxidative stress and inflammation, compared to the Aesculus hippocastanum and Vitis vinifera products.

\section{Materials and methods}

Study design. Three vegetal extracts were obtained for each plant: in water, $50 \%$ water-alcohol and in $96 \%$ ethanol. The extacts were designated as AH (for Aesculus hippocastanum), CU (for Curcuma longa) and VV (for Vitis vinifera), and indexed with w (for water), 50 (for $50 \%$ ethanol) or 96 (for $96 \%$ ethanol). Furthermore, the extracts were analyzed for their composition by HPTLC (main components) and their total phenolic content.

The extracts were further subjected to acid and alkaline hydrolysis, under mild $(0.08 \mathrm{M} \mathrm{HCl}$ and $0.02 \mathrm{M} \mathrm{NaOH})$ as well as severe conditions $(1 \mathrm{M} \mathrm{HCl}$ and $2 \mathrm{M} \mathrm{NaOH}$ under reflux) in order to examine whether the vegetal products generate hydrolytic products and alter their behavior at the gastric or at intestinal level. The hydrolysates were designated with the letters A (for acid) and B (for alkaline) hydrolysis. The in vitro antioxidant activity of the extracts, as well as that of their hydrolysates was evaluated by DPPH assay.

Jurkat lymphocytes were cultivated under normal and stress conditions (hyperglycemia mimicking diabetes mellitus) for $24 \mathrm{~h}$, and exposed to the extracts for $1 \mathrm{~h}$. They were then washed, and tested for their antioxidant activity. Cell membrane susceptibility to lipid peroxidation induced by cumene hydroperoxide (CuOOH; Sigma-Aldrich, St. Louis, MO, USA) was also examined following treatment with the extracts. The ICAM-1 expression level in the cell culture medium was measured using an ELISA kit (IBL International GmbH, Hamburg, Germany).

Plant material and extract preparation. Vitis vinifera leaves were harvested from Bicaz (Neamt County, Romania) in July 2014 and Aesculus hippocastanum seeds from Bucharest in September 2014. The plant material was dried in the dark in our laboratory $\left(25^{\circ} \mathrm{C}, 40 \%\right.$ relative humidity). Voucher specimens of the two species are available in the herbarium collection at the Department of Pharmaceutical Botany and Cell Biology, Carol Davila University of Medicine and Pharmacy, Bucharest, Romania. Curcuma longa rhizome powder was purchased from a local herb store.

A total of $15 \mathrm{~g}$ of each plant material was grounded and extracted with 3x150 ml solvent (96-ethanol 96\%; 50-ethanol-water 1:1, v:v: w-water) under reflux. The extractive solutions combined were concentrated using a rotary evaporator (RVO 004; Ingos, Prague, Czech Republic), and further dried by lyophilization at $-55^{\circ} \mathrm{C}$ (CoolSafe ScanVac 55 freeze dryer; LaboGene, Lynge, Denmark).

Hydrolysis of the extracts. In order to identify the main flavonoid aglycones, we performed acid and alkaline hydrolysis. Acid hydrolysis was performed on $100 \mathrm{mg}$ of each dry extract with $1 \mathrm{M} \mathrm{HCl}$ under reflux. Similarly, alkaline hydrolysis was performed on the same quantity from each dry extract with 
$2 \mathrm{M} \mathrm{NaOH}$ under reflux. Following hydrolysis, the flavonoid aglycones were extracted with diethyl ether. The ether was then removed using a rotary evaporator (RVO 004; Ingos), and the residue was dissolved in methanol.

Characterization of plant extracts by HPTLC/UV. The qualitative analysis of the extracts was performed by HPTLC, using HPTLC silica gel plates [Nano silica gel on TLC plates from Sigma-Aldrich Chemie GmbH (Buchs SG, Switzerland) and 60 F254 HPTLC plates from Merck Millipore (Merck KGaA, Darmstadt, Germany)] and a mobile phase consisting of toluene:ethyl acetate:formic acid 5:3:1 (v:v:v), as previously described (27). The identification of the components was performed using standard substances from Sigma-Aldrich, in two ways: by the similarity of the peak spectrum at the Rf corresponding to the standard (obtained using the facilities of TLC3 Scanner and winCATS software from Camag, Müttenz, Switzerland), and by visual observation of the peaks in UV (366 and $254 \mathrm{~nm}$ ) following derivatization with 2-aminoethyl diphenyl borate (Roth, Karlsruhe, Germany).

The amount of total phenolics in the plant extracts was determined with Folin-Ciocalteau reagent (obtained from Scharlau Co., Barcelona, Spain) using the method described in the study by González et al (28), as modified by Olaru et al (29). Briefly, to appropriate volumes of dilutions from each sample, $0.6 \mathrm{ml}$ of 1/10 dilution of Folin-Ciocalteau reagent and $2 \mathrm{ml}$ of $15 \% \mathrm{Na}_{2} \mathrm{CO}_{3}$ in water were added and incubated at $50 \pm 1^{\circ} \mathrm{C}$ for 15 min on a water bath (Memmert GmbH \& Co. KG, Schwabach, Germany). The absorbance of all samples was then measured at $765 \mathrm{~nm}$ using a Halo DB-20-220 UV/visible spectrophotometer (Dynamica, Salzburg-Mayrwies, Germany). A calibration curve using gallic acid was plotted under the same conditions. The results are expressed as micrograms of gallic acid equivalents per milligram of dry weight ( $\mu \mathrm{g}$ GAE/mg) of extract. All experiments were performed in triplicate and the means \pm standard deviation (SD) and confidence interval (95\% CI) were calculated for each sample.

Assessment of antioxidant activity of the vegetal extracts and their hydrolytic products by DPPH assay. DPPH is a stable, nitrogen-centered free radical (violet/purple color as a methanol solution). The color of DPPH gradually changes to a pale yellow (reduced form) when DPPH is exposed to free radical scavengers. The decrease in absorbance at $517 \mathrm{~nm}$ induced by antioxidants determines the reduction capability of the DPPH radicals. The advantage of this method, which makes it highly appropriate for screening the antioxidant activity of plant extracts, is the fact that it allows the testing of both lipophilic and hydrophilic compounds (30-32) and it is not restricted by the nature of the antioxidants.

As the positive controls of the experiment, ascorbic acid, quercetin and curcumin standard solutions were used. For each of these known antioxidants, the reduction of DPPH was evaluated for different concentrations of the standard: $5 \mu \mathrm{M}-1 \mathrm{mM}$ for quercetin and curcumin, and $5-250 \mu \mathrm{M}$ for ascorbic acid.

For all the prepared extracts, as well as for the products resulting from their hydrolysis, either under acidic $(1 \mathrm{M} \mathrm{HCl})$ or alkaline $(1 \mathrm{M} \mathrm{NaOH})$ conditions, the DPPH scavenging effect was assessed. For this purpose, we evaluated the decrease in the optical density of the DPPH solution (Sigma-Aldrich) mixed with each sample, for $30 \mathrm{~min}$, at $517 \mathrm{~nm}$. The results were compared with the discoloration curve of DPPH alone, as well as with the positive controls represented by ascorbic acid, curcumin and quercetin, at different levels of exposure. The antioxidant effect was expressed as the scavenged DPPH (\%) after $30 \mathrm{~min}$, when exposed to the extracts compared to the values obtained from the spontaneous discoloration of DPPH, according to the recommendations of Molyneux (32), using the following equation:

$$
\% \text { DPPH inhibition }=\frac{\mathrm{A}_{0}-\mathrm{A}_{\mathrm{s}}}{\mathrm{A}_{0}} \times 100
$$

where $A_{0}$ is the absorbance of DPPH without inhibitor and $A_{s}$ is the absorbance of the sample (DPPH plus inhibitor).

Cell cultures. Experiments were performed using Jurkat cells, from the European Collection of Cell Cultures, at the 3-4 th passage. The Jurkat lymphocytes were grown for $24 \mathrm{~h}$ in RPMI-1640 (11.11 mM glucose; Sigma-Aldrich) supplemented with $10 \%$ heat-inactivated fetal calf serum at $37^{\circ} \mathrm{C}$ in $5 \% \mathrm{CO}_{2}$ atmosphere, in 24 -well plates $\left(2 \times 10^{5}\right.$ cells/well). For diabetic conditions, the cells were plated for $24 \mathrm{~h}$ in glucose-rich medium (35 mM high glucose, equivalent to hyperglycemia associated with oxidative stress) $(33,34)$.

The prepared cells were exposed for $1 \mathrm{~h}$ to the extracts, dissolved in phosphate buffer (saline) at the concentration of $0,1 \mathrm{mg} / \mathrm{ml}$. Following exposure, the cells were washed 3 times with phosphate-buffered saline (PBS; Sigma-Aldrich), $\mathrm{pH} 7$, re-suspended in RPMI at $2 \times 10^{5}$ cells/ml and used for the assay of lipid peroxidation. Untreated cells served as negative controls and cells treated with quercetin, curcumin and ascorbic acid served as the positive controls. The viability of the cells was examined using trypan blue exlucion assay, but no significant changes were observed (data not shown).

Measurement of membrane susceptibility to lipid peroxidation. DPPP is a fluorescent probe that has weak fuorescence characteristics in its basic form; however, its oxide, which can be specifically generated in reaction with hydroperoxides, has a strong fluorescence $(34,35)$. Experimental lipid peroxidation of the cell membranes was induced treatment with $10 \mu \mathrm{M} \mathrm{CuOOH}$, generating an effective production of reactive oxygen species in the cell membrane, which was further quantified with DPPP (36).

DPPP from ThermoFisher Scientific/Invitrogen/Molecular Probes $^{\mathrm{TM}}$ (Eugene, Oregon, USA) was dissolved in dimethyl sulfoxide at a concentration of $5 \mathrm{mM}$. Following the addition of DPPP at a final concentration of $5 \mu \mathrm{M}$, the cell suspension was incubated at room temperature for $20 \mathrm{~min}$ in the dark. The DPPP-labeled cells were treated with $10 \mu \mathrm{M} \mathrm{CuOOH}$ to induce DPPP oxide formation. The process was assayed by performing fluorescence emission spectrum analysis between 360 and $410 \mathrm{~nm}$, excitation at $351 \mathrm{~nm}$, every min, for $5 \mathrm{~min}$; the fluorescence intensity maximum was measured at $380 \mathrm{~nm}$, as previously described (37) and peroxidation was computed as percentage of the fluorescent signal increase following $5 \mathrm{~min}$ of exposure to $\mathrm{CuOOH}$.

Statistical analysis. The results are expressed as the means \pm SD. Statistical analysis was performed using the Student's t-test. A value of $\mathrm{P}<0.05$ was considered to indicate a statistically significant difference. 
Table I. Results of HPTLC qualitative analysis of the extracts.

Ferrulic Caffeic Gallic p-coumaric

Extracts Esculetin acid acid acid acid Quercetin Kaempferol Chrysin Luteolin Apigenin Curcumin

A, Aesculus hippocastanum seed extracts

\begin{tabular}{|c|c|c|c|c|c|c|c|c|c|c|c|}
\hline AHwA & - & - & $?$ & - & $\mathrm{X}$ & $\mathrm{X}$ & $\mathrm{X}$ & - & - & - & - \\
\hline AHwB & - & - & $\mathrm{X}$ & - & $\mathrm{X}$ & $\mathrm{X}$ & $\mathrm{X}$ & - & - & - & - \\
\hline AH50A & - & - & - & - & - & XX & XX & - & - & $\mathrm{X}$ & - \\
\hline AH50B & - & - & - & - & - & $\mathrm{X}$ & - & - & nt & nt & $\mathrm{nt}$ \\
\hline AH96A & $\mathrm{X}$ & - & - & - & - & XXX & XXX & - & $\mathrm{nt}$ & $\mathrm{nt}$ & $\mathrm{nt}$ \\
\hline AH96B & - & - & - & - & - & $\mathrm{X}$ & - & - & nt & $\mathrm{nt}$ & $\mathrm{nt}$ \\
\hline
\end{tabular}

B, Curcuma longa rhizome extracts

\begin{tabular}{|c|c|c|c|c|c|c|c|c|c|c|c|}
\hline CUwA & - & - & - & - & - & - & - & - & - & - & - \\
\hline CUwB & - & XX & - & - & - & - & - & - & nt & $\mathrm{nt}$ & XX \\
\hline CU50A & - & - & - & - & - & - & $?$ & $?$ & - & - & $\mathrm{XXX}$ \\
\hline CU50B & - & XX & - & - & - & - & - & - & $\mathrm{nt}$ & $\mathrm{nt}$ & $\mathrm{X}$ \\
\hline CU96A & - & - & - & - & - & - & $?$ & $?$ & - & - & XXX \\
\hline CU96B & - & XXX & $?$ & - & - & - & - & - & - & - & $\mathrm{X}$ \\
\hline
\end{tabular}

C, Vitis vinifera leaf extracts

\begin{tabular}{lccccccccccc}
\hline VVwA & $\mathrm{X}$ & - & $\mathrm{X}$ & - & - & $\mathrm{XXX}$ & $?$ & - & $\mathrm{nt}$ & $\mathrm{nt}$ & $\mathrm{nt}$ \\
VVwB & - & - & $\mathrm{XX}$ & - & $?$ & $\mathrm{XX}$ & - & - & - & - & - \\
VV50A & $\mathrm{X}$ & - & $\mathrm{X}$ & - & - & $\mathrm{XX}$ & - & - & $\mathrm{nt}$ & $\mathrm{nt}$ & $\mathrm{nt}$ \\
VV50B & - & - & $\mathrm{XX}$ & $\mathrm{X}$ & $?$ & $\mathrm{X}$ & - & - & $\mathrm{X}$ & - & - \\
VV96A & $\mathrm{XXX}$ & - & $\mathrm{XX}$ & - & - & $\mathrm{XX}$ & - & - & $\mathrm{nt}$ & $\mathrm{nt}$ & $\mathrm{nt}$ \\
VV96B & - & $?$ & $\mathrm{XX}$ & - & $?$ & $\mathrm{X}$ & - & - & $\mathrm{X}$ & - & -
\end{tabular}

HPTLC, high performance thin layer chromatography; X, present; XX, present - intense; XXX, present - very intense; ?, possibly present; nt, not tested; -, absent; AH, Aesculus hippocastanum; CU, Curcuma longa; VV, Vitis vinifera; w, water extract; 50, 50\% ethanol extract; 96, 96\% ethanol extract; A, hydrolysis products after $1 \mathrm{M} \mathrm{HCl}$ reflux; B, hydrolysis products after $\mathrm{NaOH}$ reflux.

\section{Results and Discussion}

Chemical characterization of the total extracts. HPTLC analysis performed on the extracts revealed the presence of certain polyphenols and flavones in the extracts, as presented in Table I.

The results obtained from the analysis of the total flavonoids are presented in Table II. All relative standard deviations (RSD) were $<5 \%$.

The main flavonoids and phenolic acids identified in the three extracts were as follows: p-coumaric acid, caffeic acid, quercetin, kaempferol, apigenin and esculetin (Aesculus hippocastanum); ferrulic acid and curcumin (Curcuma longa); caffeic acid, gallic acid, quercetin, luteolin and esculetin (Vitis vinifera). Besides, in the case of Curcuma longa and Vitis vinifera a few other tested standard substances presented positive results: caffeic acid, kaempferol and chrysisn, and ferrulic acid, p-coumaric acid and kaemferol, respectively. However, due to the fact that the UV-VIS spectra (data not shown) of the spots supposed to belong to the above-mentioned substances were not similar to the corresponding standards (possibly due to other unidentified components that co-eluted with the searched
Table II. Quantitative analysis of total phenolic compounds present in the extracts.

\begin{tabular}{lccc}
\hline & \multicolumn{3}{c}{ Total flavonoids $(\mu \mathrm{g} / \mathrm{mg}$ total extract $)$} \\
\cline { 2 - 4 } Extract & Mean & SD & $95 \%$ CI \\
\hline AHw & 0.386 & 0.0058 & $0.379-0.393$ \\
AH50 & 0.756 & 0.0111 & $0.729-0.785$ \\
AH96 & 1.358 & 0.0421 & $1.291-1.425$ \\
CUw & 9.475 & 0.3113 & $8.702-10.250$ \\
CU50 & 39.182 & 0.6810 & $37.490-40.870$ \\
CU96 & 38.326 & 0.9992 & $35.840-40.810$ \\
VVw & 1.140 & 0.0195 & $1.092-1.189$ \\
VV50 & 2.496 & 0.0543 & $2.362-2.632$ \\
VV96 & 4.220 & 0.0232 & $4.163-4.279$ \\
\hline
\end{tabular}

$\mathrm{AH}$, Aesculus hippocastanum; $\mathrm{CU}$, Curcuma longa; $\mathrm{VV}$, Vitis vinifera; w, water extract; 50,50\% ethanol extract; $96,96 \%$ ethanol extract; SD, standard deviation, CI, confidence interval. 
Table III. Antioxidant effects of positive controls expressed as a percentage of scavenged DPPH.

\begin{tabular}{|c|c|c|c|c|c|}
\hline \multicolumn{2}{|c|}{ Curcumin } & \multicolumn{2}{|c|}{ Quercetin } & \multicolumn{2}{|c|}{ Ascorbic acid } \\
\hline $\begin{array}{l}\text { Concentration } \\
(\mathrm{mM})\end{array}$ & $\begin{array}{c}\text { DPPH inhibition } \\
(\%)\end{array}$ & $\begin{array}{c}\text { Concentration } \\
(\mathrm{mM})\end{array}$ & $\begin{array}{c}\text { DPPH inhibition } \\
(\%)\end{array}$ & $\begin{array}{l}\text { Concentration } \\
(\mathrm{mM})\end{array}$ & $\begin{array}{c}\text { DPPH inhibition } \\
(\%)\end{array}$ \\
\hline 0.005 & 3.673 & 0.005 & 2.635 & 0.005 & 1.846 \\
\hline 0.01 & 5.635 & 0.01 & 6.846 & 0.025 & 9.635 \\
\hline 0.05 & 13.692 & 0.05 & 14.75 & 0.050 & 18.077 \\
\hline 0.1 & 21.692 & 0.1 & 29.327 & 0.125 & 46.211 \\
\hline 0.2 & 26.038 & 0.2 & 54.077 & 0.250 & 85.423 \\
\hline 0.5 & 44.788 & 0.5 & 85.269 & - & - \\
\hline 1 & 76.404 & - & - & - & - \\
\hline
\end{tabular}

DPPH, 2,2-diphenyl-1-picrylhydrazyl.

Table IV. Antioxidant effect exerted by the extracts, expressed as a percentage of scavenged DPPH.

\begin{tabular}{lccc}
\hline Extract & $\begin{array}{c}\text { Non-hydrolyzed } \\
\text { extract }\end{array}$ & $\begin{array}{c}\text { Acid } \\
\text { hydrolysis (A) }\end{array}$ & $\begin{array}{c}\text { Alkaline } \\
\text { hydrolysis (B) }\end{array}$ \\
\hline AHw & -6.235 & 31.173 & 20.121 \\
AH50 & 14.066 & 1.323 & 6.367 \\
AH96 & 1.323 & -6.830 & 22.832 \\
CUw & 22.312 & 78.358 & 26.715 \\
CU50 & 37.257 & 47.941 & 24.296 \\
CU96 & 53.797 & 81.768 & 24.882 \\
VVw & 43.633 & 83.195 & 23.512 \\
VV50 & 53.816 & 85.330 & 21.396 \\
VV96 & 72.974 & 62.073 & 28.651 \\
\hline
\end{tabular}

DPPH, 2,2-diphenyl-1-picrylhydrazyl; AH, Aesculus hippocastanum; CU, Curcuma longa; VV, Vitis vinifera; w, water extract; 50, 50\% ethanol extract; 96, 96\% ethanol extract; A, hydrolysis products after $1 \mathrm{M}$ $\mathrm{HCl}$ reflux; $\mathrm{B}$, hydrolysis products after $\mathrm{NaOH}$ reflux.

phenolic compounds) we decided to mark them as 'possibly present' (Table I). Other major spots with a positive reaction for phenolic compounds (data not shown) were observed in all the extracts, but they were not identified on the basis of a standard substance.

The highest yield of total flavonoids from horse chestnut seeds and common grape wine leaves was obtained by extraction with $96 \%$ ethanol and was approximately 3.5-fold higher than the content obtained by extraction with water. With the extraction of turmeric with 96 and $50 \%$ ethanol, we obtained close values with similar upper limits and slightly different lower limits of $95 \%$ CI. The use of ethanol and hydro-ethanol for the extraction of turmeric resulted in a total phenolic content yield of approximately 4-fold higher compared to extraction with water (Table II).

In vitro antioxidant effect assessed by DPPH assay. The kinetics of DPPH decoloration for various concentrations of positive
Table V. Antioxidant effects induced by the prepared extracts, expressed as the susceptibility to lipid peroxidation evaluated through a percentage increase in DPPP due to DPPP oxide generation.

\begin{tabular}{lcc}
\hline & \multicolumn{2}{c}{ DPPP fluorescence increase $(\%)$} \\
\cline { 2 - 3 } Treatment of the cells & $\begin{array}{c}\text { Normoglycemic } \\
\text { growth medium }\end{array}$ & $\begin{array}{c}\text { Hyperglycemic } \\
\text { growth medium }\end{array}$ \\
\hline Control (untreated cells) & 166.205 & 180.607 \\
Curcumin 10 $\mu \mathrm{M}$ & 134.967 & 90.159 \\
Quercetin 10 $\mu \mathrm{M}$ & 112.026 & 81.346 \\
Ascorbic acid 10 $\mu \mathrm{M}$ & 121.892 & 88.727 \\
AHw & 164.052 & 140.477 \\
AH50 & 162.412 & 135.538 \\
AH96 & 169.935 & 133.857 \\
CUw & 150.279 & 93.945 \\
CU50 & 134.808 & 82.639 \\
CU96 & 118.037 & 59.344 \\
VVw & 158.421 & 106.181 \\
VV50 & 117.139 & 108.442 \\
VV96 & 60.711 & 96.578
\end{tabular}

DPPP, diphenyl-1-pyrenylphosphine; AH, Aesculus hippocastanum; CU, Curcuma longa; VV, Vitis vinifera; w, water extract; 50, 50\% ethanol extract; 96, 96\% ethanol extract.

controls was tested before the main measurements to establish the reagent concentration and the speed of the reaction (data not shown). We finally found that within $30 \mathrm{~min}$, the reaction was completed (DPPH was scavenged), and the results obtained for the positive controls (curcumin, quercetin and ascorbic acid) are presented in Table III. The results ranged from $2 \%$ scavenged DPPH (lowest concentration of quercetin and ascorbic acid tested) to $85 \%$ scavenged DPPH (highest concentration of quercetin or ascorbic acid tested). It is interesting that ascorbic acid and quercetin are more potent antioxidants compared with curcumin. 

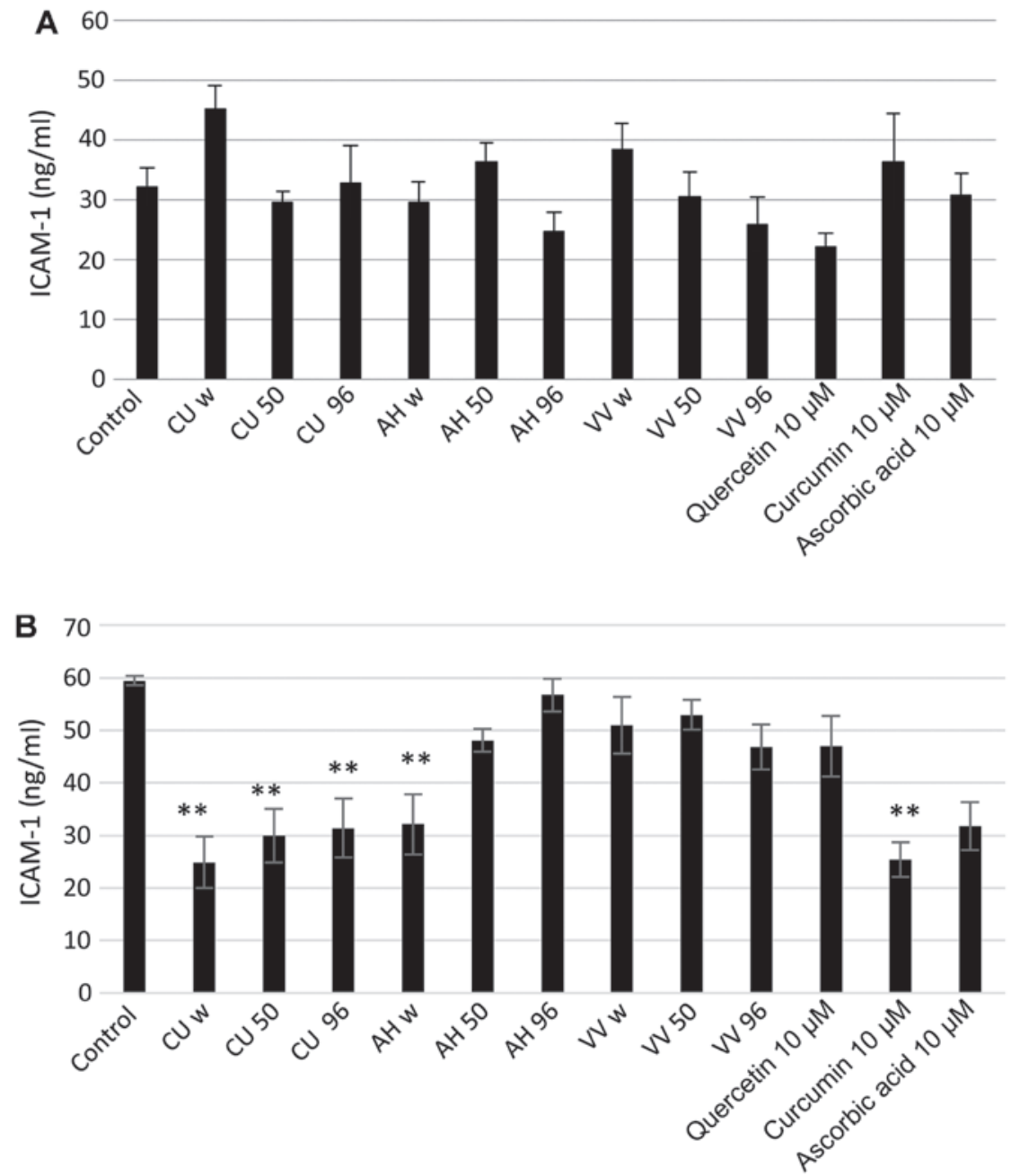

Figure 1. Intercellular adhesion molecule-1 (ICAM-1) expression level in the growth medium of cells grown in (A) normal glucose (11.11 mM) medium and treated with vegetal extracts or (B) in high glucose $(35 \mathrm{mM})$ medium and treated with vegetal extracts. AH, Aesculus hippocastanum; CU, Curcuma longa; $\mathrm{VV}$, Vitis vinifera; w, water extract; 50, 50\% ethanol extract; $96,96 \%$ ethanol extract. ${ }^{*} \mathrm{p}<0,05,{ }^{* *} \mathrm{p}<0,001$.

Furthermore, the ability of the extracts and that of their hydrolisates to scavenge DPPH was evaluated. Our results revealed that, in the case of the Aesculus hippocastanum extracts, the antioxidant effects were limited. The discoloration kinetics for the sample solutions (data not shown) was close to that of DPPH alone; therefore, the ability of the extracts to reduce DPPH radicals was rather insignificant.

The aqueous and $96 \%$ ethanol extracts did not exhibit antioxidant effects; however, such effects were observed for the 50\% ethanol extract. As regards the effects exerted by the hydrolytic products of the Aesculus hippocastanum extracts, the results revealed that the acid, as well as the alkaline hydrolysis of the aqueous extracts and the alkaline hydrolysis products of the $96 \%$ ethanol extract exhibited antioxidant effects (Table IV).

The antioxidant activity of the Curcuma longa extracts increased with the increase in the ethanol concentration of the extraction solvent. The alkaline hydrolysis resulted in mixtures with a low antioxidant activity (approximately 20\%), but the acid hydrolysis generated a high antioxidnat activity from all the extracts (Table IV).

The antioxidant effects induced by the Vitis vinifera extracts increased with the increase in the ethanolic concentration of the extraction solvent. The alkaline hydrolysis generated, from all the extracts, products with the same type of antioxidant activity (approximately 20-30\% inhibition of DPPH). The antioxidant activity of the products resulting from acid hydrolysis was supperior $(>60 \%)$ in the case of all extracts (Table IV).

Antioxidant and anti-inflammatory effects exerted by the vegetal extracts in a cell culture model of diabetes mellitus. In order to extrapolate the results into in vivo models, the antioxidant effects of the extracts were assessed in a model of Jurkat cells, cultivated either in RPMI medium with $11.11 \mathrm{mM}$ glucose (normoglycemic) or in RPMI medium supplemented with glucose up to $35 \mathrm{mM}$ (equivalent with hyperglycemia and oxidative stress). A low susceptibility to lipid peroxidation (\%) expressed a greater protective effect against hydroperoxide generation.

The exposure of the cells to the extracts did not lead to a reduction in cell viability (data not shown) in both models. The results also revealed that the Aesculus hippocastanum extract did not prove effective in reducing the generation of membrane lipoperoxides; these results confirmed the effects obtained with the DPPH experiments. The antioxidant effects of the Vitis vinifera extracts were higher compared to those exerted by 
the Curcuma longa extracts, but only for the extracts prepared in alcoholic medium. It should be noted that the reduction in hydroperoxide generation was greater in the extracts prepared with an increased concentration of ethanol, for the normoglycemia cell model.

For cells exposed to hyperglycemia and oxidative stress, the susceptibility to lipid peroxidation in the control sample was greater compared to the one obtained with $11.11 \mathrm{mM}$ glucose medium, as prevoiusly shown (34). The efficacy of the Vitis vinifera and Curcuma longa extracts was in the same range as that observed for the positive controls (quercetin, curcumin and ascorbic acid). The antioxidant efficacy of the Curcuma long extrats was significantly greater compared to that of all the other extracts in the hyperglycemic medium (Table V).

The results demonstrated that, under normal glucose conditions, there were no significant changes in the level of ICAM-1 in the medium (Fig. 1A). Under hyperglycemic conditions, the level of ICAM-1 in the untreated cells was higher compared to the cells in normal glucose medium (Fig. 1B). All the tested Curcuma longa extracts induced a significant decrease in ICAM-1 expression in the cell medium, similar to that induced by the standard curcumin $(10 \mu \mathrm{M})$ solution. The Aesculus hippocastanum and Vitis vinifera extracts had limited anti-inflammatory effects.

In conclusion, in the present study, we prepared three types of extracts (in water, 50\% ethanol and $96 \%$ ethanol) from three different vegetal products: Aesculus hippocastanum (semen), Vitis vinifera L. folium (both indigenous plants in Romania) and Curcuma longa L. rhizoma, available on the Romanian market.

The obtained extracts had different phenolic contents, depending on the plant and type of extract, ranging from 0.4 to $39 \mu \mathrm{g}$ phenolics/mg total extract. The greatest amount of phenolics was found in the Cucurma longa extracts, while the lowest, in the Aesculus hippocastanum extacts. HPTLC analysis revealed the following components in the extracts: quercetin, kaempferol, coumaric acid, caffeic acid, apigenin and esculetin in the Aesculus hippocastanum extracts; ferrulic acid and curcumin in the Cucuma longa extracts; and quercetin, ferrulic acid and gallic acid, kaempferol, luteolin, and esculetin in the Vitis vinifera extacts.

The evaluation of hydrolisis resistance proved that, for all the extracts, no significant chemical changes were obtained under mild hydrolisis conditions (artificial gastric juice and artificial intestinal fluid).

The antioxidant effects of the extracts and their hydrolitic products were assessed by DPPH assay. The results revealed that the Aesculus hippocastanum extracts had a low antioxidant efficacy. Both the Curcuma longa and Vitis vinifera extracts had a high antioxidant activity; the products resulting from alkaline hydrolisis were significantly more efficient in scavenging DPPH radicals compared to their acid hydrolitic products.

The antioxidant effects induced by the extracts on the cell membrane were evaluated as the susceptibility to lipid peroxidation in two different models: Jurkat cells grown in normal glucose $(11.11 \mathrm{mM})$ and high glucose $(35 \mathrm{mM})$ medium. The activity of the Curcuma long extracts was significantly greater under both experimental conditions. The Vitis vinifera extracts also proved to be effective in normoglycemic medium, but not under hyperglycemic conditions.
Exposure to hyperglycemic conditions induced an increase in the ICAM-1 level compared to normal glucose conditions. All the tested Curcuma longa extracts induced a significant decrease in ICAM-1 expression in the cell medium, similar to that induced by the standard curcumin $(10 \mu \mathrm{M})$ solution. The Aesculus hippocastanum and Vitis vinifera extracts had limited anti-inflammatory effects.

These results could be translated into clinical practice and demonsrated that Curcuma longa extracts may be effective both in the prevention of diabetes mellitus and in attenuating the associated complications, particularly cardiovascular complications.

\section{Acknowledgements}

The present study was performed in the framework of the Global Research Initiative 2014 action financed by Bionorica Germany.

\section{References}

1. Fraga CG, Galleano M, Verstraeten SV and Oteiza PI: Basic biochemical mechanisms behind the health benefits of polyphenols. Mol Aspects Med 31: 435-445, 2010.

2. DayoubO, Andriantsitohaina R and Clere N: Pleiotropic beneficial effects of epigallocatechin gallate, quercetin and delphinidin on cardiovascular diseases associated with endothelial dysfunction. Cardiovasc Hematol Agents Med Chem 11: 249-264, 2013.

3. Camer D, Yu Y, Szabo A and Huang XF: The molecular mechanisms underpinning the therapeutic properties of oleanolic acid, its isomer and derivatives for type 2 diabetes and associated complications. Mol Nutr Food Res 58: 1750-1759, 2014.

4. Hubbard BP and Sinclair DA: Small molecule SIRT1 activators for the treatment of aging and age-related diseases. Trends Pharmacol Sci 35: 146-154, 2014.

5. Chen J, Mangelinckx S, Adams A, Wang ZT, Li WL and De Kimpe N: Natural flavonoids as potential herbal medication for the treatment of diabetes mellitus and its complications. Nat Prod Commun 10: 187-200, 2015.

6. Sen T and Samanta SK: Medicinal plants, human health and biodiversity: a broad review. Adv Biochem Eng Biotechnol 147: 59-110, 2015.

7. EMA/HMPC/16635/2009 Committee on Herbal Medicinal Products (HMPC): Community herbal monograph on Vitis vinifera L., folium, July 15, 2010. http://www.ema.europa.eu/ docs/en_GB/document_library/Herbal_-_Community_herbal_ monograph/2011/01/WC500100391.pdf. Accessed August 29, 2015.

8. EMA/HMPC/354156/2011 Committee on Herbal Medicinal Products (HMPC): Community herbal monograph on Aesculus hippocastanum L., cortex, May 22, 2012. http://www.ema.europa. eu/docs/en_GB/document_library/Herbal_-_Community_herbal_ monograph/2012/06/WC500129249.pdf. Accessed August 29, 2015.

9. EMA/HMPC/456845/2008, corr. Committee on Herbal Medicinal Products (HMPC): Community herbal monograph on Curcuma longa L., rhizoma, November 6, 2008. http://www.ema.europa.eu/ docs/en_GB/document_library/Herbal___Community_herbal monograph/2009/12/WC500018230.pdf. Áccessed August 29, $201 \overline{5}$.

10. Hasegawa T, Nakatani K, Fujihara T and Yamada H: Aroma of turmeric: Dependence on the combination of groups of several odor constituents. Nat Prod Commun 10: 1047-1050, 2015.

11. Schmidt E, Ryabchenko B, Wanner J, Jäger W and Jirovetz L: Cytotoxic active constituents of essential oils of Curcuma longa and Curcuma zanthorrhiza. Nat Prod Commun 10: 139-141, 2015.

12. Li Y, Li J, Li S, Li Y, Wang X, Liu B, Fu Q and Ma S: Curcumin attenuates glutamate neurotoxicity in the hippocampus by suppression of ER stress-associated TXNIP/NLRP3 inflammasome activation in a manner dependent on AMPK. Toxicol Appl Pharmacol 286: 53-63, 2015.

13. Vallianou NG, Evangelopoulos A, Schizas N and Kazazis C: Potential anticancer properties and mechanisms of action of curcumin. Anticancer Res 35: 645-651, 2015. 
14. Hong SL, Lee GS, Syed Abdul Rahman SN, Ahmed Hamdi OA, Awang K, Aznam Nugroho N and Abd Malek SN: Essential oil content of the rhizome of Curcuma purpurascens Bl. (Temu Tis) and its antiproliferative effect on selected human carcinoma cell lines. ScientificWorld Journal 2014: 397430, 2014.

15. Cao Y, Xu RX and Liu Z: A high-throughput quantification method of curcuminoids and curcumin metabolites in human plasma via high-performance liquid chromatography/tandem mass spectrometry. J Chromatogr B Analyt Technol Biomed Life Sci 949-950: 70-78, 2014.

16. Villaflores OB, Chen YJ, Chen CP, Yeh JM and Wu TY: Curcuminoids and resveratrol as anti-Alzheimer agents. Taiwan J Obstet Gynecol 51: 515-525, 2012.

17. Ahmed T and Gilani AH: Therapeutic potential of turmeric in Alzheimer's disease: curcumin or curcuminoids? Phytother Res 28: 517-525, 2014

18. Jiang JL, Li ZD, Zhang H, Li Y, Zhang XH, Yuan YF and Yuan YJ: Feature selection for the identification of antitumor compounds in the alcohol total extracts of Curcuma longa. Planta Med 80: 1036-1044, 2014.

19. Vecchi Brumatti L, Marcuzzi A, Tricarico PM, Zanin V, Girardelli $\mathrm{M}$ and Bianco AM: Curcumin and inflammatory bowel disease: potential and limits of innovative treatments. Molecules 19: 21127-21153, 2014.

20. Dudek-Makuch M and Matławska I: Flavonoids from the flowers of Aesculus hippocastanum. Acta Pol Pharm 68: 403-408, 2011.

21. Orhan DD, Orhan N, Ozcelik B and Ergun F: Biological activities of Vitis vinifera L. leaves. Turk J Biol 33: 341-348, 2009.

22. Fernandes F, Ramalhosa E, Pires P, Verdial J, Valentão P, Andrade P, Bento A and Pereira JA: Vitis vinifera leaves towards bioactivity. Ind Crops Prod 43: 434-440, 2013.

23. Ríos-Hoyo A, Cortés MJ, Ríos-Ontiveros H, Meaney E, Ceballos G and Gutiérrez-Salmeán G: Obesity, metabolic syndrome, and dietary therapeutical approaches with a special focus on nutraceuticals (polyphenols): a mini-review. Int J Vitam Nutr Res 84 113-123, 2014

24. Fuentes E and Palomo I: Mechanisms of endothelial cell protection by hydroxycinnamic acids. Vascul Pharmacol 63: 155-161, 2014.

25. Elliott L and Cifu AS: Healthy lifestyle counseling in persons with cardiovascular risk factors. JAMA 314: 398-399, 2015.

26. Sarriá B, Martínez-López S, Sierra-Cinos JL, Garcia-Diz L, Goya L, Mateos R and Bravo L: Effects of bioactive constituents in functional cocoa products on cardiovascular health in humans. Food Chem 174: 214-218, 2015.
27. Wagner $\mathrm{H}$ and Bladt S (eds): Plant Drug Analysis: A Thin Layer Chromatography Atlas. 2nd edition. Springer-Verlag, Berlin, p197, pp286-288, 1996.

28. González M, Guzmán B, Rudyk R, Romano E and Molina MAA: Spectrophotometric determination of phenolic compounds in propolis. Lat. Am. J. Pharm. 22: 243-248, 2003.

29. Olaru O, Şeremet OC, Petrescu M, Sălăgean A, Velescu B and Niţulescu M: Toxicity evaluation and polyphenols assessment of some extracts from indigenous Euphorbia species. Rom. J. Biopys. 24: 43-54, 2014.

30. Kumkrai P, Weeranantanapan $\mathrm{O}$ and Chudapongse $\mathrm{N}$ : Antioxidant, $\alpha$-glucosidase inhibitory activity and sub-chronic toxicity of Derris reticulata extract: Its antidiabetic potential. BMC Complement Altern Med 15: 35, 2015.

31. Iqbal D, Khan MS, Khan MS, Ahmad S, Hussain MS and Ali M Bioactivity guided fractionation and hypolipidemic property of a novel HMG-CoA reductase inhibitor from Ficus virens Ait. Lipids Health Dis 14: 15, 2015.

32. Molyneux P: The use of the stable free radical diphenylpicrylhydrazyl (DPPH) for estimating antioxidant activity. Songklanakarin J Sci Technol 26: 211-219, 2004.

33. Margina D, Gradinaru D, Manda G, Neagoe I and Ilie M: Membranar effects exerted in vitro by polyphenols - quercetin, epigallocatechin gallate and curcumin - on HUVEC and Jurkat cells, relevant for diabetes mellitus. Food Chem Toxicol 61: 86-93, 2013.

34. Margina D, Ilie M, Manda G, Neagoe I, Danciulescu-Miulescu R, Purdel CN and Gradinaru D: In vitro effects of prolonged exposure to quercetin and epigallocatechin gallate of the peripheral blood mononuclear cell membrane. Cell Mol Biol Lett 19: 542-560, 2014.

35. Gomes A, Fernandes E and Lima JLFC: Fluorescence probes used for detection of reactive oxygen species. J Biochem Biophys Methods 65: 45-80, 2005.

36. Takahashi M, Shibata M and Niki E: Estimation of lipid peroxidation of live cells using a fluorescent probe, diphenyl-1-pyrenylphosphine. Free Radic Biol Med 31: 164-174, 2001.

37. Okimotoa Y, Watanabea A, Nikia E, Yamashitab $T$ and Noguchia N: A novel fluorescent probe diphenyl-1-pyrenylphosphine to follow lipid peroxidation in cell membranes. FEBS Lett 474: 137-140, 2000. 